\title{
DIFFERENTIATION WITH RESPECT TO A FUNCTION OF LIMITED
}

\section{VARIATION *}

BY

P. J. DANIELL

Lebesgue, Radon, and Young $\dagger$ have defined integrals with respect to a function of limited variation, and these are generalizations of the Stieltjes integral. The next step which suggests itself is a definition of the corresponding derivative. Such a definition is given in this paper, and the fundamental property of a derivative is proved by means of a modification of Vitali's theorem. The steps taken are parallels of steps given by de la Vallee Poussin $\ddagger$ in the theory of Lebesgue plane sets. Since this paper was first written, a paper by Young§ has appeared, giving a slightly different definition of the derivative, and an entirely different trea ment. The applications at the end of this paper are not given by Young.

Definition of derived numbers and derivative. Consider two functions of $x, F(x), \alpha(x)$, defined in the fundamental interval, $0 \leqq x \leqq 1$. The ratio

$$
\frac{F(x+)-F(x-\epsilon)}{\alpha(x+\epsilon)-\alpha(x-\epsilon)}=\frac{\Delta F}{\Delta \alpha}
$$

may have upper and lower limits as $\epsilon$ approaches 0 . We define these as the upper and lower derived numbers of $F(x)$ with resp ct to $\alpha(x)$, and we use the notation

$$
\bar{D}_{a} F(x)=\varlimsup_{\epsilon=0} \frac{\Delta F}{\Delta \alpha}, \quad \underline{D}_{a} F(x)=\varliminf_{e \leq 0} \frac{\Delta F}{\Delta \alpha} .
$$

For $x$ equal to 0 or 1 it is necessary to add a convention whereby $F(x)$, $\alpha(x)$ are continued beyond the range $(0,1)$, and have values equal to their values at 0 or 1 respectively. If the two derived numbers are finite and equal,

* Presented to the Society, September 6, 1918.

$\dagger$ Lebesgue, C o m p te s R e nd u s, vol. 150 (1910), p. 86; Radon, Wien e r Sitzu n g s berichte, vol. 122, section 2a (1913), p. 1295; W. H. Young, Proce e ding s of the London Mathematical Society, vol. 13 (1914), p. 109.

$\ddagger$ De la Vallée Poussin, these T r a n s a c t i o n s, vol. 16 (1915), p. 435.

\$W. H. Young, Proceedings of the London Mathematical Society, vol. 15 (1916), p. 35. 
that is, if the relative change ratio $\Delta F / \Delta \alpha$ possesses a finite limit, this limit is called the $\alpha$-derivative of $F(x)$, and is denoted by

$$
D_{a} F(x) \text {. }
$$

An interval $(x-\epsilon, x+\epsilon)$ we shall call a central interval with center at $x$.

If $\alpha(x), F(x)$, are functions of limited variation, we can define absolutely additive functions of sets measurable Borel

$$
\int_{E} d \alpha, \quad \int_{E} d F
$$

and also the corresponding modular integrals, denoted according to Radon by

$$
\int_{E}|d \alpha|, \quad \int_{E}|d F|
$$

or according to the writer by

$$
\int_{E} d \omega, \quad \int_{E} d \Omega
$$

where $\omega(x), \Omega(x)$, are the variation functions corresponding to $\alpha(x), F(x)$. These modular integrals are additive, finite, and non-negative.

Absolute continuity. A function $F(x)$ is said to be absolutely continuous relative to $\alpha(x)$, a function of limited variation, if given any positive $\epsilon$ we can find $\delta$ so that

$$
\int_{e}^{0} d \Omega(x)<\epsilon
$$

for all sets $e$ measurable Borel such that

$$
\int_{e} d \omega(x)<\delta
$$

where $\omega(x), \Omega(x)$ are the variation functions corresponding to $\alpha(x), F(x)$. We desire to prove the fundamental proposition:

Theorem. If $F(x)$ is absolutely continuous relative to $\alpha(x)$, it possesses a finite $\alpha$-derivative nearly everywhere ( $\omega)$ [that is, except for a point set $e$ for which $\int_{c} d \omega(x)=0$ ]; this $\alpha$-derivative is summable $(\alpha)$ where it exists, and if $E$ is any set measurable Borel,

$$
\int_{E} d F(x)=\int_{E} D_{\alpha} F(x) d \alpha(x) .
$$

In this, $D_{\alpha} F(x)$ denotes the $\alpha$-derivative of $F(x)$ where it exists, and any finite value where it does not. 
Before we can prove this proposition, it is necessary to prove two lemmas.

Lemma 1. Given any positive $\epsilon$ and a non-decreasing function $\omega(x)$, any set $E$ measurable Borel can be enclosed strictly in a finite or denumerable system $A$ of disjoint interrals (that is, intervals with no points common to any two) in su:h a way that

$$
\int_{A} d \omega<\int_{E} d \omega+\epsilon
$$

Let $D$ be the set of points at which, $\omega(x)$ has finite discontinuities; then $D$ consists at most of a denumerable set of points. Resolve $\omega(x)$ into $\omega_{1}(x)$, a continuous non-decreasing function, and $\omega_{2}(x)$, a non-decreasing function which is stationary except when $x$ passes a point of $D$. The integral $\int_{E} d \omega_{1}(x)$ will be a continuous additive non-negative function of sets. Hence, ${ }^{*}$ given any positive $\epsilon$, we can enclose $E$ strictly in a finite or denumerable system $A_{1}$ of disjoint intervals so that

$$
\int_{A_{1}} d \omega_{1}(x)<\int_{E} d \omega_{1}(x)+\frac{1}{2} \epsilon .
$$

The set $C E \cdot D$ consists at most of a denumerable set of points $D^{\prime}$. Then, since

$$
\int_{D^{\prime}} d \omega(x)=\int_{D^{\prime}} d \omega_{2}(x)
$$

is a convergent series of positive terms (considered as the sum of the discontinuities of $\omega(x)$ at the denumerable set of points $D^{\prime}$ ), we can choose a finite $n$ and the finite set of points $D_{n}$ so that

$$
\int_{D^{\prime}} d \omega_{2}(x)<\int_{D_{n}} d \omega_{2}(x)+\frac{1}{2} \epsilon .
$$

From $A_{1}$ cut out the points belonging to $D_{n}$, which are finite in number. Then $A=A_{1} \cdot C D_{n}$ still forms a denumerable system of intervals enclosing $E$ strictly; for the points $D_{n}$ belong to $C E$. The set $A$ is the same as $A_{1}$ except for the exclusion of a finite set of points, or

$$
\int_{A} d \omega_{1}(x)=\int_{A_{1}} d \omega_{1}(x)<\int_{E} d \omega_{1}(x)+\frac{1}{2} \epsilon .
$$

Again, from the way in which $D_{n}$ was chosen,

But

$$
\int_{C E \cdot D \cdot C D_{n}} d \omega_{2}(x)<\frac{1}{2} \epsilon .
$$

$$
\int_{e} d \omega_{2}(x)=0
$$

\footnotetext{
* De la Vallée Poussin, loc. cit., p. 470.
} 
over any set $e$ not containing points of $D$. Hence

$$
\int_{C E \cdot c D_{n}} d \omega_{2}(x)<\frac{1}{2} \epsilon, \quad \int_{A_{1} \cdot c E \cdot c D_{n}} d \omega_{2}(x)<\frac{1}{2} \epsilon,
$$

and, since $A=A_{1} \cdot C D_{n}$,

Hence

$$
\int_{A \cdot C E} d \omega_{2}(x)<\frac{1}{2} \epsilon, \quad \int_{A} d \omega_{2}(x)<\int_{E} d \omega_{2}(x)+\frac{1}{2} \epsilon .
$$

$$
\int_{\Lambda} d \omega(x)=\int_{A} d \omega_{1}(x)+\int_{A} d \omega_{2}(x)<\int_{E} d \omega(x)+\epsilon .
$$

The lemma is proved.

The following lemma is a generalization of Vitali's theorem.*

Lemma 2. Given a set $E$ measurable Borel, and an infinite family $\Gamma$ of central intervals, such that each point of $E$ is the center of an infinity of central intervals as small as we please; then a set $B$ can be found consisting of a finite or denumerable number of disjoint intervals chosen from $\Gamma$, such that $B$ covers nearly all $E$ (that is, except for a point set of w-measure 0 ) and such that he $\omega$-measure of $B$ differs fiom that of $E$ by as little as we please.

That is to say, given any positive $\epsilon$, we can find $B$ so that

$$
\int_{E \cdot C B} d \omega(x)=0, \quad \int_{B} d \omega(x)<\int_{E} d \omega(x)+\epsilon .
$$

By means of Lemma 1, given any positive $\epsilon$, we can enclose $E$ strictly in a denumerable set of disjoint intervals $A$, so that

$$
\int_{A} d \omega^{\prime}<\int_{E} d \omega^{\prime}+\epsilon,
$$

where $\omega^{\prime}(x)=\omega(x)+x$. In what follows we denote the $\omega^{\prime}$-measure of a set $E$ simply by $m E$. Then $m E$ is the sum of the $\omega$-measure and the usual Lebesgue measure. The intervals $A$ are not necessarily central intervals. From the family $\Gamma$ eliminate the intervals which have points in common with $C A$. The remaining family $\Gamma_{1}$ will possess the same property relative to $E$, for each point of $E$ is interior (strictly) to one or other of the intervals $A$.

We affirm that with a finite number of disjoint intervals of $\Gamma_{1}$ we can cover a part $e_{1}$ of $E$ such that $m e_{1}>k m E$, where $k$ is any number less than one-third. For $\dagger$ let the set $C E$ be enclosed strictly in a denumerable set $D$ of open intervals so that $m D$ is arbitrarily close to $m C E$. Then $C D$ is a closed set contained in $E$, and given any positive $\epsilon$ we can make $m C D>m E-\epsilon$. By the Heine-

* See de la Vallée Poussin, Cours d'Analyse, second edition, vol. 2, p. 110.

$\dagger$ This part of the proof is due to the Editors of these Transactions. 
Borel Theorem* $C D$ can be covered by a finite number of intervals $E_{n}$ chosen from the family $\Gamma_{1}$. Then

$$
m E_{n} \geqq m C D>m E-\epsilon .^{*}
$$

From these intervals choose first that one having the greatest $\omega^{\prime}$-measure, and e'iminate those which have any point in common with it. Next choose the remaining interval which has the greatest $\omega^{\prime}$-measure, and so on. After a finite number of such steps we shall have chosen a finite number of intervals, and the process will terminate. Each time intervals are eliminated, the $\omega^{\prime}$-measure of the interval retained will be at least one-third of the $\omega^{\prime}$-measure of the interval covered by it and all intervals eliminated as overlapping it. Hence the measure of all intervals retained will be at least $\frac{1}{3} m E_{n}$, or will be greater $\operatorname{than} \frac{1}{3} m E-\epsilon$. The part of $E$ not covered, that is $E-e_{1}$, is therefore of measure less than $m A-\frac{1}{3} m E+\epsilon$, or less than $\frac{2}{3} m E+2 \epsilon$. Hence

$$
m e_{1}>\frac{1}{3} m E-2 \epsilon \text {. }
$$

Our affirmation is proved, that with a finite number of disjoint intervals belonging to $\Gamma_{1}$ we can cover a part $e_{1}$ of $E$ so that

$$
m e_{1}>k m E \text {, }
$$

where $k$ is any number less than one-third.

After we have thus chosen $e_{1}$, omit from $\Gamma_{1}$ those intervals which overlap $e_{1}$, and let $\Gamma_{2}$ be the remainder. Then $\Gamma_{2}$ will have the same properties relative to $E-e_{1}$ as $\Gamma_{1}$ has relative to $E$. We can by the same process cover a portion $e_{2}$ of $E-e_{1}$, such that

$$
m e_{2}>k m\left(E-e_{1}\right),
$$

by a finite number of intervals of $\Gamma_{1}$. Continuing the process, we obtain a system $B$ of disjoint intervals of the family $\Gamma$, which are at most denumerable. Moreover the set $B \cdot E=\sum e_{n}$. But

$$
m e_{n}>k\left(m E-m e_{1}-\cdots-m e_{n-1}\right),
$$

and the term in the parenthesis is non-negative, and therefore $\sum m e_{n}$ is convergent. Thus $m e_{n}$ approaches zero, or

Then

$$
m\left(\sum e_{n}\right)=\sum m e_{n}=m E
$$

$$
m E \cdot B=m E, \quad m E \cdot C B=0, \quad \int_{E \cdot C B} d \omega^{\prime}=0 .
$$

But $\omega^{\prime}(x)=\omega(x)+x$, so that

* Cf. de la Vallée Poussin, Intégrales de Lebesgue, pp. 13-15. 
and, since

$$
\int_{E \cdot C B} d \omega \leqq \int_{E \cdot C B} d \omega^{\prime}=0
$$

$$
\int_{A-E} d \omega \leqq \int_{A-E} d \omega^{\prime}<\epsilon, \quad \int_{A} d \omega<\int_{E} d \omega+\epsilon .
$$

But $B$ is contained in $A$, or

The lemma is proved.

$$
\int_{B} d \omega<\int_{E} d \omega+\epsilon
$$

To return to our original proposition, let us prove it first in the case where $\alpha(x)$ is a non-decreasing function, so that $\alpha(x)=\omega(x)$.

In any set $e$, if $\bar{D}_{\omega} F(x) \geqq l$ (this inequality being considered to hold if $\left.\bar{D}_{\omega} F(x)=+\infty\right)$, we shall show that

$$
\int_{e} d F \geqq l \int_{e} d \omega
$$

When this is proved, it will follow as a corollary, and can also be established directly by parallel reasoning, that a similar conclusion holds if the signs of inequality are reversed, or if $\bar{D}$ is replaced by $\underline{D}$, or both. At any rate, it is sufficient to give the proof for the case first mentioned. Corresponding to every point in $e$ (which is measurable Borel) we can find an infinity of central intervals as small as we please, such that for each

$$
\frac{\Delta F}{\Delta \omega}>l-\epsilon^{\prime}
$$

given any positive $\epsilon^{\prime}$. These form a family $\Gamma$ having the Vitali property relative to $e$. Since $F(x)$ is absolutely continuous with respect to $\omega(x)$, given any positive $\epsilon$ we can find $\delta$ so that

for all sets $e$ for which

$$
\int_{e} d \Omega<\epsilon,
$$

$$
\int_{e} d \omega<\delta
$$

Using Lemma 2, we can define a set $B$ consisting of a denumerable system of disjoint intervals belonging to $\Gamma$, such that

whence

$$
\int_{e \cdot C B} d \omega=0
$$

$$
\int_{e \cdot C B} d \Omega=n
$$


and such that

whence

$$
\int_{B \cdot C_{e}} d \omega<\delta
$$

Then

$$
\int_{B \cdot C e} d \Omega<\epsilon
$$

$$
\left|\int_{e} d \omega-\int_{B} d \omega\right|<\delta, \quad\left|\int_{e} d F-\int_{B} d F\right|<\epsilon .
$$

But for each of the intervals $B$,

$$
\begin{gathered}
\frac{\Delta F}{\Delta \omega}>l-\epsilon^{\prime}, \quad \Delta F>l \Delta \omega-\epsilon^{\prime} \Delta \omega, \quad \int_{B} d F>l \int_{B} d \omega-\epsilon^{\prime} \int_{B} d \omega, \\
\int_{e} d F>\left(l-\epsilon^{\prime}\right) \int_{e} d \omega-\epsilon-|l| \delta-\epsilon^{\prime} \delta .
\end{gathered}
$$

In the limit, as $\epsilon, \epsilon^{\prime}$ approach $0, \delta$ also approaches 0 , and

$$
\int_{e} d F \geqq l \int_{e} d \omega \text {. }
$$

Since $\int_{e} d F$ is limited, the $\omega$-measure of $e$ decreases to the limit 0 as $l$ increases indefinitely. Also, since $F$ is absolutely continuous with respect to $\omega$, $\int_{e} d F$ will also approach the limit 0 . Thus the set of points for which $\bar{D}_{\omega} F(x)=+\infty$ is of $\omega$-measure 0 . A similar proof shows that the set for which $\underline{D}_{\omega} F(x)=-\infty$ is of $\omega$-measure 0 .

Take two finite numbers $m, M$, and divide the interval between them into sub-intervals by $m=l_{0}<l_{1}<\cdots<l_{n}=M$, where $\max \left|l_{i}-l_{i-1}\right|<$ a given positive $\eta$. Let $e_{i}$ be the set in $E$ for which $l_{i-1} \leqq \bar{D}_{\omega} F<l_{i}$; then

$$
\begin{aligned}
& l_{i-1} \int_{e_{i}} d \omega \leqq \int_{e_{i}} d F \leqq l_{i} \int_{e_{i}} d \omega, \\
& \left|\int_{e_{i}} d F-l_{i} \int_{e_{i}} d \omega\right| \leqq \eta \int_{e_{i}} d \omega .
\end{aligned}
$$

By summing up for the sets $e_{i}$, if $E^{\prime}$ is the set of points where $m \leqq \bar{D}_{\omega} F<M$,

$$
\left|\int_{E^{\prime}} d F-\sum_{i} l_{i} \int_{e_{i}} d \omega\right| \leqq \eta \int_{E^{\prime}} d \omega
$$

in the limit, as $\eta$ approaches $0, \bar{D}_{\omega} F$ is summable $(\omega)$ in $E^{\prime}$, and

$$
\int_{E^{\prime}} d F=\int_{E^{\prime}} \bar{D}_{\omega} F d \omega
$$


It has been proved already that $\int_{E-E^{\prime}} d F$ approaches 0 as $M$ increases and $m$ decreases indefinitely, whence $\bar{D}_{\omega} F$ is summable $(\omega)$ in $E$, and

$$
\int_{E} d F=\int_{E} \bar{D}_{\omega} F d \omega
$$

Similarly it can be proved that $\underline{D}_{\omega} F$ is summable $(\omega)$ in $E$, and

$$
\int_{E} d F=\int_{E} \underline{D}_{\omega} F d \omega
$$

But $\underline{D}_{\omega} F \leqq \bar{D}_{\omega} F$, or at every point of $E$ except on a point set of $\omega$-measure 0 ,

$$
\underline{D}_{\omega} F=\bar{D}_{\omega} F=D_{\omega} F,
$$

and $F(x)$ has a finite derivative with respect to $\omega(x)$ nearly everywhere $(\omega)$ in $E \quad$ Also

$$
\int_{E} d F=\int_{E} D_{\omega} F d \omega
$$

More generally, if $\alpha(x)$ is a function of limited variation, it is absolutely continuous with respect to its variation function $\omega(x)$. We may split any set $E$ measurable Borel into two subsets $E_{1}, E_{2}$, so that if $e$ is a variable set,

$$
\int_{e \cdot E_{1}} d \alpha=\int_{e \cdot E_{1}} d \omega
$$

a non-negative function of sets, and

$$
\int_{e \cdot E_{2}} d \alpha=-\int_{e \cdot E_{2}} d \omega \text {. }
$$

We have already proved that

$$
\int_{E_{1}} d F=\int_{E_{1}} D_{\omega} F d \omega=\int_{E_{1}} D_{\omega} F d \alpha,
$$

and by the same proof $D_{\omega} \alpha$ exists and is finite nearly everywhere $(\omega)$, and

$$
\int_{e \cdot E_{1}} d \omega=\int_{e \cdot E_{1}} d \alpha=\int_{e \cdot E_{1}} D_{\omega} \alpha d \omega
$$

whence $D_{\omega} \alpha=\lim \Delta \alpha / \Delta \omega$ exists and equals 1 nearly everywhere $(\omega)$ in $E_{1}$. It follows that $D_{a} F$ exists and equals $D_{\omega} F$ nearly everywhere $(\omega)$ in $E_{1}$; or

$$
\int_{E_{1}} d F=\int_{E_{1}} D_{a} F d \alpha
$$

Similarly in $E_{2}, D_{a} F$ exists and equals $-D_{\omega} F$ nearly everywhere $(\omega)$, and 


$$
\int_{E_{2}} d F=\int_{E_{2}} D_{\omega} F d \omega=-\int_{E_{2}} D_{\omega} F d \alpha=\int_{E_{2}} D_{a} F d \alpha .
$$

By combining $E_{1}$ and $E_{2}$, it is proved that $D_{a} F$ exists and is finite in $E$ except for a set of $\omega$-measure 0 , that it is summable in $E$, and that

$$
\int_{E} d F(x)=\int_{E} D_{a} F(x) d \alpha(x) .
$$

Applications. Reduction of general integral to integral of positive type. Let $g(x)$ denote the function

$$
g(x)=D_{\omega} \alpha(x),
$$

where this derivative $=+1$ or -1 , and

$$
g(x)=0
$$

otherwise, that is to say on a set of $\omega$-measure 0 . On the set $E_{1}, g(x)=1$ nearly everywhere $(\omega)$, whence, if $f(x)$ is any function summable $(\omega)$,

$$
\int_{E_{1}} f(x) d \alpha(x)=\int_{E_{1}} f(x) d \omega(x)=\int_{E_{1}} f \dot{(x) g(x) d \omega(x) .}
$$

Similarly,

whence

$$
\int_{E_{2}} f(x) d \alpha(x)=-\int_{E_{2}} f(x) d \omega(x)=\int_{E_{2}} f(x) g(x) d \omega(x),
$$

$$
\int_{E} f(x) d \alpha(x)=\int_{E} f(x) g(x) d \omega(x) .
$$

This proves that any integral with respect to a function of bounded variation $\alpha(x)$ can be expressed as a single integral of positive type, that is to say, an integral with respect to a non-decreasing function $\omega(x)$.

Integration by Parts. Let $F(x)=\alpha^{2}(x)$, where $\alpha(x)$ is a function of limited variation. The latter function may be expressed as the difference of two that are non-decreasing,

whence

$$
\alpha(x)=\beta_{1}(x)-\beta_{2}(x),
$$

$$
\begin{aligned}
F(x)=\alpha^{2}(x) & =\beta_{1}^{2}(x)+\beta_{2}^{2}(x)-2 \beta_{1}(x) \beta_{2}(x), \\
\Omega(x) & =\beta_{1}^{2}(x)+\beta_{2}^{2}(x)+2 \beta_{1}(x) \beta_{2}(x)=\omega^{2}(x) .
\end{aligned}
$$

The function $\omega(x)$ is limited and less than some finite number $K$, so that for any interval

$$
\Delta \Omega<2 K \Delta \omega ;
$$


whence $F(x)=\alpha^{2}(x)$ is absolutely continuous with respect to $\alpha(x)$. In this case

$$
\frac{\Delta F}{\Delta \alpha}=\frac{\alpha^{2}(x+\epsilon)-\alpha^{2}(x-\epsilon)}{\alpha(x+\epsilon)-\alpha(x-\epsilon)}=\alpha(x+\epsilon)+\alpha(x-\epsilon) .
$$

Let $\bar{\alpha}(x)$ denote

then

$$
\lim _{\epsilon=0} \frac{1}{2}[\alpha(x+\epsilon)+\alpha(x-\epsilon)] ;
$$

$$
D_{a} \alpha^{2}(x)=2 \bar{\alpha}(x) \text {. }
$$

By the fundamental proposition,

$$
\int_{E} d \alpha^{2}(x)=\int_{E} 2 \bar{\alpha}(x) d \alpha(x) .
$$

Let $\alpha_{1}(x), \alpha_{2}(x)$ be two functions of limited variation; then

$$
\begin{aligned}
\int d\left[\left(\alpha_{1}+\alpha_{2}\right)^{2}\right] & =\int d \alpha_{1}^{2}+\int d \alpha_{2}^{2}+2 \int d\left(\alpha_{1} \alpha_{2}\right) \\
& =2 \int\left(\bar{\alpha}_{1}+\bar{\alpha}_{2}\right) d\left(\alpha_{1}+\alpha_{2}\right) \\
& =2 \int \bar{\alpha}_{1} d \alpha_{1}+2 \int \alpha_{2} d \alpha_{2}+2 \int \bar{\alpha}_{1} d \alpha_{2}+2 \int \bar{\alpha}_{2} d \alpha_{1} .
\end{aligned}
$$

So

$$
\int_{E} d\left(\alpha_{1} \alpha_{2}\right)=\int_{E} \bar{\alpha}_{1} d \alpha_{2}+\int_{E} \bar{\alpha}_{2} d \alpha_{1}
$$

Transposed and written more fully, the last relation becomes

$$
\int_{E} \bar{\alpha}_{1}(x) d \alpha_{2}(x)=\int_{E} d\left[\alpha_{1}(x) \alpha_{2}(x)\right]-\int_{E} \bar{\alpha}_{2}(x) d \alpha_{1}(x) .
$$

Rice Institute,

Houston, Texas. 